\title{
Improved Gabor Deconvolution and Its Extended Applications
}

\author{
Xuekai SUN and Sam Zandong SUN \\ Lab for Integration of Geology and Geophysics (LIGG), \\ College of Geophysics and Information Engineering, \\ China University of Petroleum (Beijing), Beijing, China \\ e-mails: xksun_paper@126.com (corresponding author), szd@cup.edu.cn
}

\begin{abstract}
In log time-frequency spectra, the nonstationary convolution model is a linear equation and thus we improved the Gabor deconvolution by employing a log hyperbolic smoothing scheme which can be implemented as an iteration process. Numerical tests and practical applications demonstrate that improved Gabor deconvolution can further broaden frequency bandwidth with less computational expenses than the ordinary method. Moreover, we attempt to enlarge this method's application value by addressing nonstationary and evaluating $Q$ values. In fact, energy relationship of each hyperbolic bin (i.e., attenuation curve) can be taken as a quantitative indicator in balancing nonstationarity and conditioning seismic traces to the assumption of unchanging wavelet, which resultantly reveals more useful information for constrained reflectivity inversion. Meanwhile, a statistical method on $Q$-value estimation is also proposed by utilizing this linear model's gradient. In practice, not only estimations well agree with geologic settings, but also applications on $Q$-compensation migration are favorable in characterizing deep geologic structures, such as the pinch-out boundary and water channel.
\end{abstract}

Key words: Gabor deconvolution, nonstationary, hyperbolic smoothing, log spectra, $Q$-compensation migration.

Ownership: Institute of Geophysics, Polish Academy of Sciences;

(C) 2015 Sun and Sun. This is an open access article distributed under the Creative Commons Attribution-NonCommercial-NoDerivs license,

http://creativecommons.org/licenses/by-nc-nd/3.0/. 


\section{INTRODUCTION}

Unlike stationary deconvolution, Gabor deconvolution is based on a nonstationary convolution, which is a pseudo-differential operator containing explicit representations of the attenuation function and thus allows the wavelet to evolve with time (Margrave 1998, Margrave and Lamoureux 2001). Usually, Gabor deconvolution separates the reflectivity by estimating the source wavelet and attenuation under the assumption of white-reflectivity and minimum-phased wavelet (Margrave et al. 2011). Due to the consideration of attenuation, Gabor deconvolution is able to achieve better resolution than stationary deconvolution types, especially for deep reservoirs. More importantly, Gabor deconvolution is more capable in addressing the nonstationarity of seismograms than either time-varying deconvolution or inverse- $Q$ filtering, mainly because the time-varying deconvolution is sensitive to the empirical parameters (e.g., window size) (Griffiths et al. 1977, Koehler and Taner 1985) and inverse- $Q$ filtering might suffer from instability (Bickel and Natarajan 1985, Hargreaves and Calvert 1991, Wang 2002). In the last decade, scholars devoted energy to make Gabor deconvolution to be more reliable and robust by seeking suitable window functions, robust spectrum estimations, accurate phase corrections and better smoothing methods (Grossman et al. 2002, Margrave et al. 2003, Montana and Margrave 2005, $\mathrm{Yu}$ and Liu 2012, Chen et al. 2013), and all these improvements have contributed its popularity in seismic processing.

In this paper, we try to find a more efficient realization for Gabor deconvolution in log time-frequency spectra as the way like homomorphic deconvolution (Ulrych 1971, Tria et al. 2007). More importantly, we also concentrate our energy on enlarging and deepening the applications of this technique in seismic data processing by balancing the intrinsic nonstationarity of seismic traces and estimating variant- $Q$ values from a different perspective, in which the former challenge the popular assumption of unchanging wavelet for most routine inversion methods and the latter are urgently required for many useful techniques in dealing with attenuation, such as inverse- $Q$ filter and $Q$-compensation migration.

\section{THEORY AND NUMERICAL TESTS}

With the help of Gabor transform (Margrave et al. 2004), the nonstationary trace $\hat{s}_{g}(t, f)$ can be factorized as (Margrave 1998)

$$
\hat{s}_{g}(t, f) \approx \hat{w}(f) \alpha(t, f) \hat{r}_{g}(t, f),
$$

where $\hat{w}(f)$ is time-independent source wavelet, and $\hat{r}_{g}(t, f)$ represents the Gabor transform of reflectivity. What is more, $\alpha(t, f)$ denotes the attenuation function with minimum-phased delay 


$$
\alpha(t, f)=e^{-\pi f t / Q+i H(\pi f t / Q)} .
$$

Here, $Q$ is the quality factor of attenuating medium, and $H$ denotes the Hilbert transform. Only considering the magnitude of Eq. 1, we get

$$
\left|\hat{s}_{g}(t, f)\right| \approx|\hat{w}(f)||\alpha(t, f)|\left|\hat{r}_{g}(t, f)\right| \text {. }
$$

In words, Eq. 3 represents the nonstationary convolution model in Gabor domain which forms the basis of Gabor deconvolution in many literatures (Margrave et al. 2002, Montana and Margrave 2005). Yet, Eq. 3 takes on a linear form by doing log transform

$$
L_{\hat{s}_{g}}=\frac{-\pi}{Q} t f+\left[L_{\hat{w}}(f)+L_{\hat{r}_{g}}(t, f)\right],
$$

where $L$ stands for logarithm operation on its subscript. Major improvements of above equation lie in that it cast some new lights on the factorization of nonstationary convolution model, because its gradient is closely related to $Q$ while the intercept is caused by the source and reflectivity.

To enable a better separation, we introduce a log hyperbolic smoothing scheme here (Sun et al. 2013). In the first step, the $t-f \log$ spectra need to be divided into numbers of hyperbolic bins. Figure 1a illustrates a partition of 20 hyperbolic bins on a numerical model. Then, we get an attenuation curve by investigating the root-mean-square (i.e., RMS) values of all the bins. For cases free of attenuation, this curve is basically horizontal even for a practical blue reflectivity sequence. Actually, the attenuation can be recovered through the linearity of the curve, while source wavelet is then approached
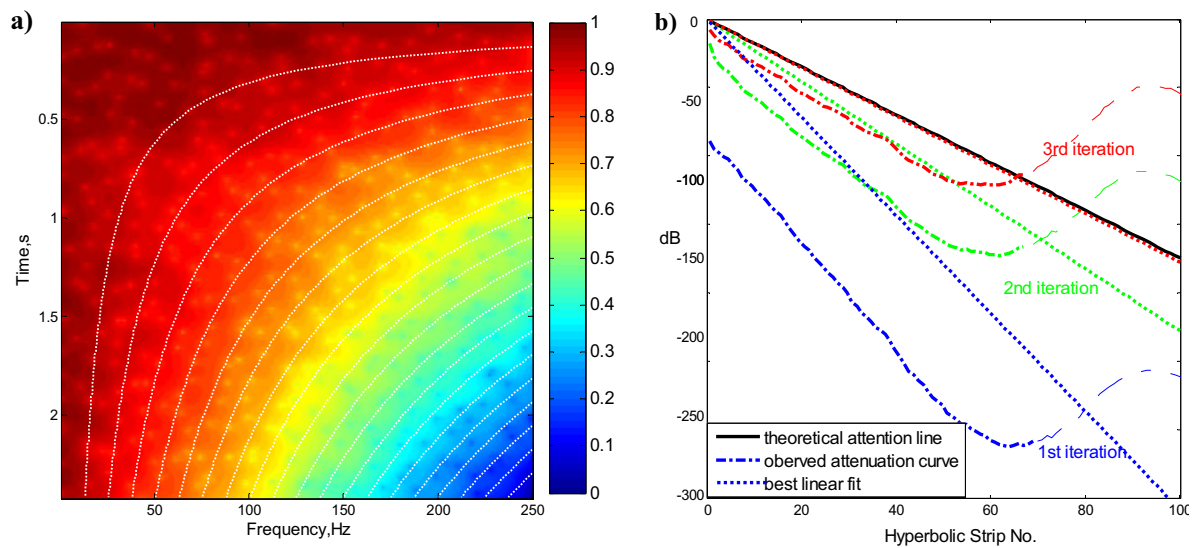

Fig. 1: (a) Twenty hyperbolic bins in log spectra, and (b) the iteration process for purifying the estimation of attenuation (blue - the 1st iteration, green - the 2nd iteration, red - the 3rd iteration). 
by averaging over the time direction and smoothing along the frequency direction (Margrave et al. 2011). Yet, the first estimation of attenuation can hardly be reliable due to the influence of source. It is evident that the existence of source wavelet usually causes an overestimated attenuation, which leads to an underestimated source in return. For this reason, we recommend an iteration process in purifying attenuation by eliminating the influence of source wavelet gradually. From another perspective, the iterative process proceeds to seek better estimations on attenuation and source wavelet until the attenuation curve is almost balanced. More importantly, the improved Gabor deconvolution shows remarkable accuracy and efficiency, because the separation in log spectra is largely done by subtractions instead of division, thus evading the usage of pre-whitening factors on denominator.

For a better illustration of the improvements, we use a famous 3-layerreflectivity model (Margrave et al. 2003), where a weak reflectivity zone is surrounded by two normal ones. In this model, attenuated synthetic is generated by employing a minimum-phase source with a dominant frequency of $40 \mathrm{~Hz}$ and a $Q$ value of 32 . With this model, we compare the performances of 3 different smoothing methods, namely, boxcar smoothing, ordinary hyperbolic smoothing, and log hyperbolic smoothing. The ordinary hyperbolic smoothing and log hyperbolic smoothing are the kernel of conventional and improved type of Gabor deconvolution, respectively. In Gabor transform, the Gaussian window is set to be 32-points, with a 8-point-long moving step along the time axis. The whole spectra are divided into 100 hyperbolic partitions for these two types of hyperbolic smoothing. Meanwhile, in boxcar smoothing, the size of boxcar is selected to be $10 \mathrm{~Hz}$ along the frequency axis and 1/5 length of the trace length along the time axis. Deconvolution results in Gabor-transformed domain are given in Fig. 2. Obviously, magnitude recovered by log hyperbolic smoothing is almost identical with actual response. In comparison, boxcar smoothing tends to smear the energy differences between different zones, while ordinary hyperbolic smoothing might unreasonably boost or suppress some areas' energy. Considering that boxcar smoothing is prone to cause AGC effects, it is seldom adopted by nowadays Gabor deconvolution.

Additionally, we attach great importance on the efficiency of iteration process, which not only works to eliminate the influence of source and refine attenuation estimation, but also determines the overall computational expenses. To measure iteration efficiency, a residual source ratio $R$ is defined as

$$
R(i)=\frac{\left\|\hat{w}_{i}(f)\right\|_{2}}{\left\|\hat{w}_{1}(f)\right\|_{2}} \times 100 \%, \quad i \geq 2,
$$



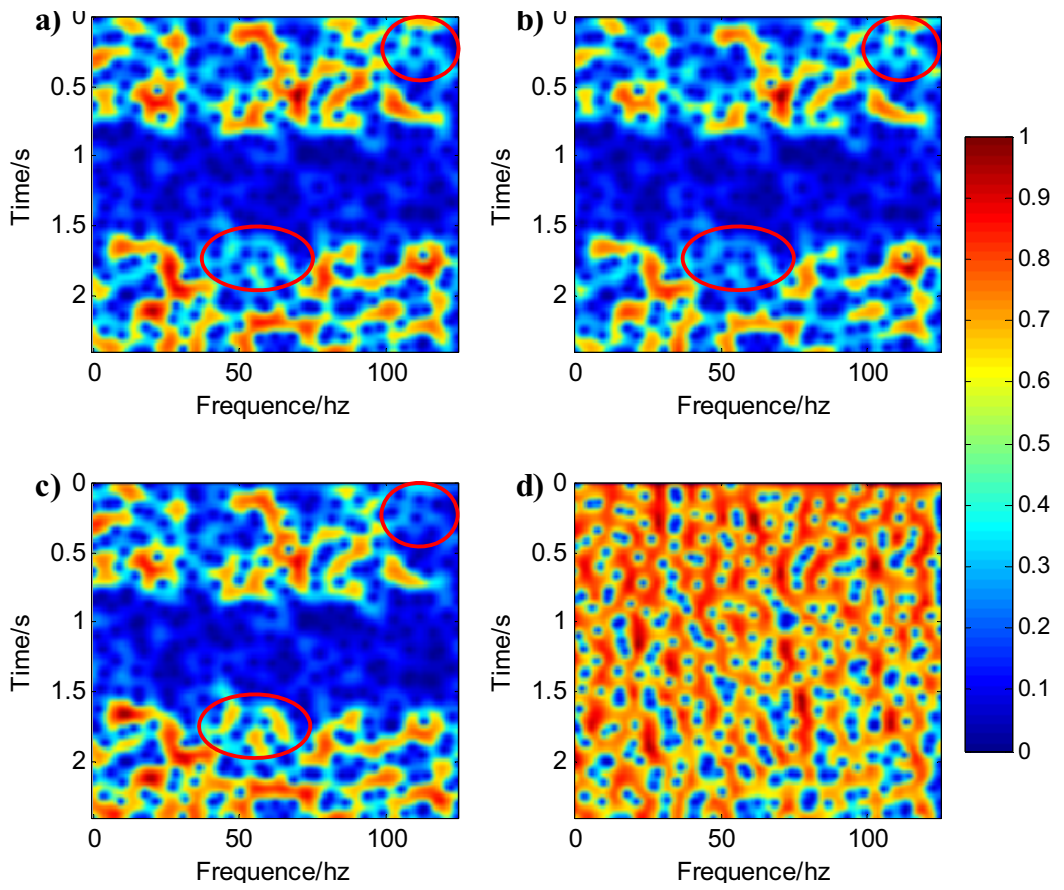

Fig. 2. Magnitude spectrum generated from 3 different smoothing methods: (a) actual Gabor magnitude spectrum, (b) by log hyperbolic smoothing, (c) by ordinary hyperbolic smoothing, and (d) by boxcar smoothing.

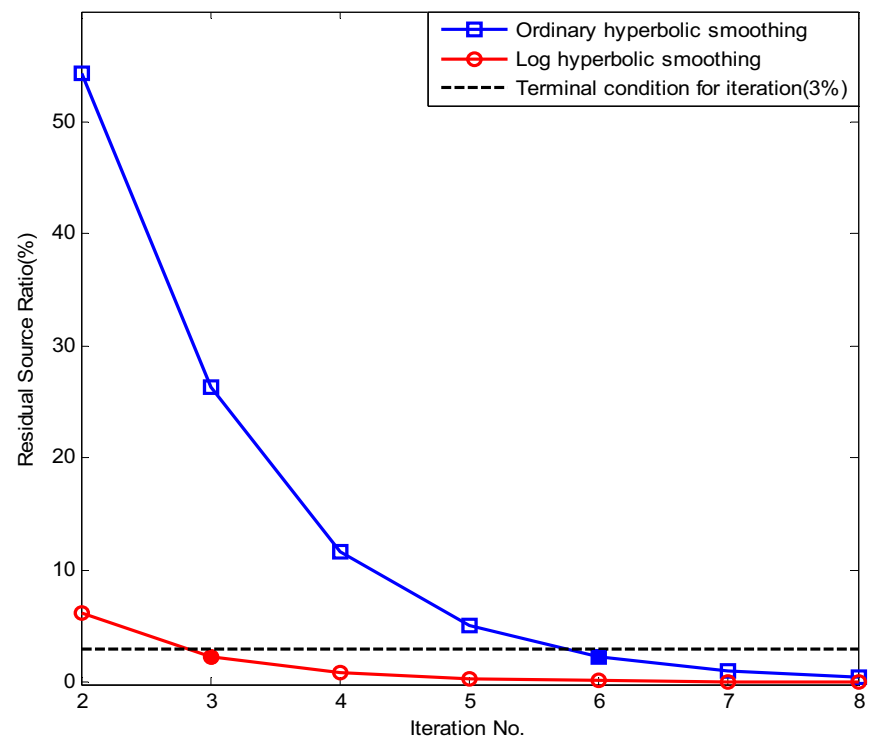

Fig. 3. Iterative efficiency comparisons for the 2 hyperbolic smoothing methods. 
where $\|\cdots\|_{2}$ stands for $l_{2}$-norm. $\hat{w}_{1}(f)$ denotes the first estimation of wavelet which is taken as a fixed standard to quantity how much the residual source remains in subsequent iterations. The smaller the ratio, the more efficient it proves. As Fig. 3 shows, $R$ quickly reaches the terminal condition $(R<3 \%)$ after 3 iterations for log hyperbolic smoothing, compared with 6 iterations required by ordinary hyperbolic smoothing.

\section{APPLICATIONS ON RESOLUTION-ENHANCEMENTS}

At present, the industry commonly uses Gabor deconvolution on enhancing seismic resolution. In this example, we first inspect resolution improvements prompted by this improved Gabor deconvolution on a migrated 2D seismic line across a potential reef reservoir. Many reflectors exist inside the reef but seldom show themselves on conventional imaging section (see Fig. 4a) because of the tuning effects. Comparisons of Gabor deconvolution results and their corresponding frequency spectra are given in Fig. 4. Smoothing method in time-frequency is always regarded as the key for Gabor deconvolution, since it actually employs an iteration process in factorizing the nonstationary convolutional model and thus estimating the reflectivity. Using ordinary hyperbolic smoothing, we derive a deconvolution result in Fig. 4c, which
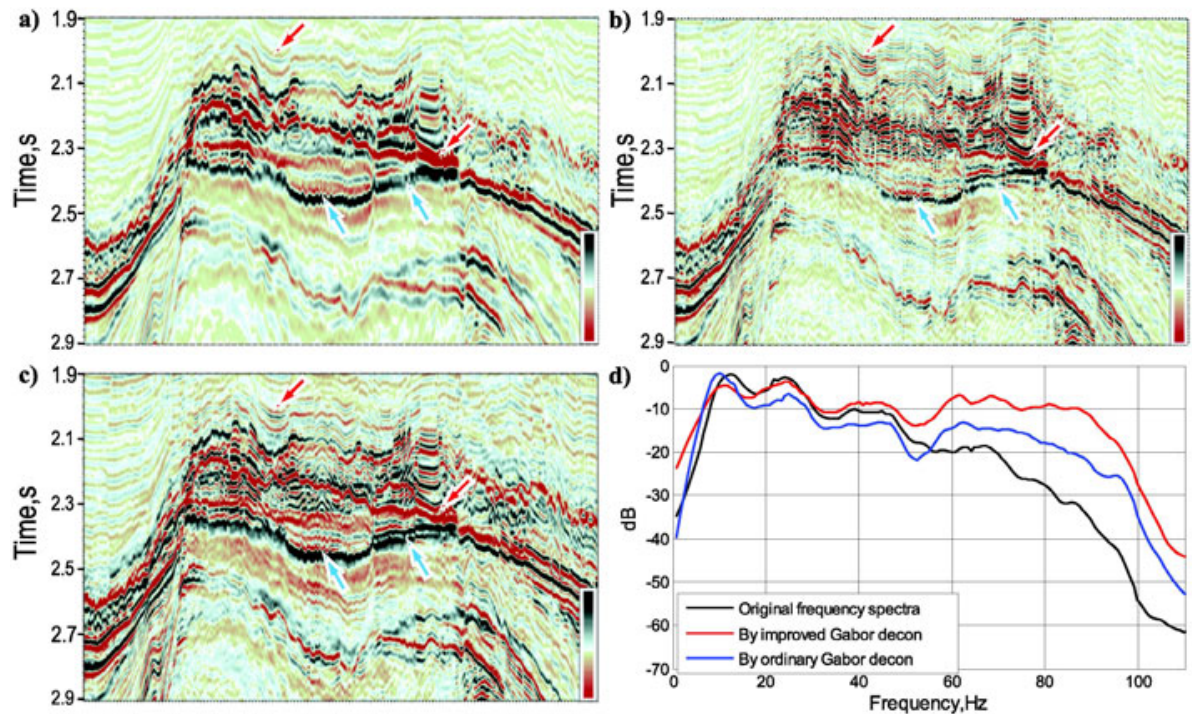

Fig. 4. Gabor deconvolution results on a reef reservoir with different smoothing methods: (a) original seismic section, (b) result by improved Gabor deconvolution, (c) result by conventional Gabor deconvolution, and (d) corresponding frequency spectra. It shows that the improved Gabor deconvolution reveals more detailed reflections inside the reef, and better decomposes the tuning effects, compared with the conventional method. 
shows a relatively improved seismic resolution. However, due to pre-whitening factors during iteration, this conventional Gabor deconvolution is not sufficient enough to reveal detailed reflections inside, while the tuning effects at the bottom is not obviously decomposed. By contrast, result from the improved Gabor deconvolution finally reveals a much more detailed structure of this reef (see Fig. 4b), in which many subtle reflections (marked by the red arrows) are obviously enhanced and the bottom tuning effect (marked by the blue arrows) is also better decomposed. With the help of the improved method, the frequency bandwidth is further broadened and high frequency energy is boosted, when compared with that of traditional method.

\section{APPLICATIONS ON BALANCING NONSTATIONARITY}

As a matter of fact, the improved Gabor deconvolution can cast some new lights on addressing the issue of nonstationarity, to which attenuation is generally acknowledged to be a major contributor. Using the improved Gabor deconvolution, we would proceed to discuss how to quantify and correct influence of nonstationarity on reflectivity estimation. As Fig. 5a shows, the study target is shallow simple strata that is formed under a relatively stable sedimentary situation. To illustrate the existence of nonstationarity, we analyze the time-frequency spectra of one seismic trace and find that dominant frequency of the top half is higher and the frequency band is also broader than that of the bottom half (see Fig. 5b). In fact, it is a common phenomenon that challenges the basic assumption of unchanging wavelet for most inversion methods which are built on Robinson convolution (Robinson 1954).
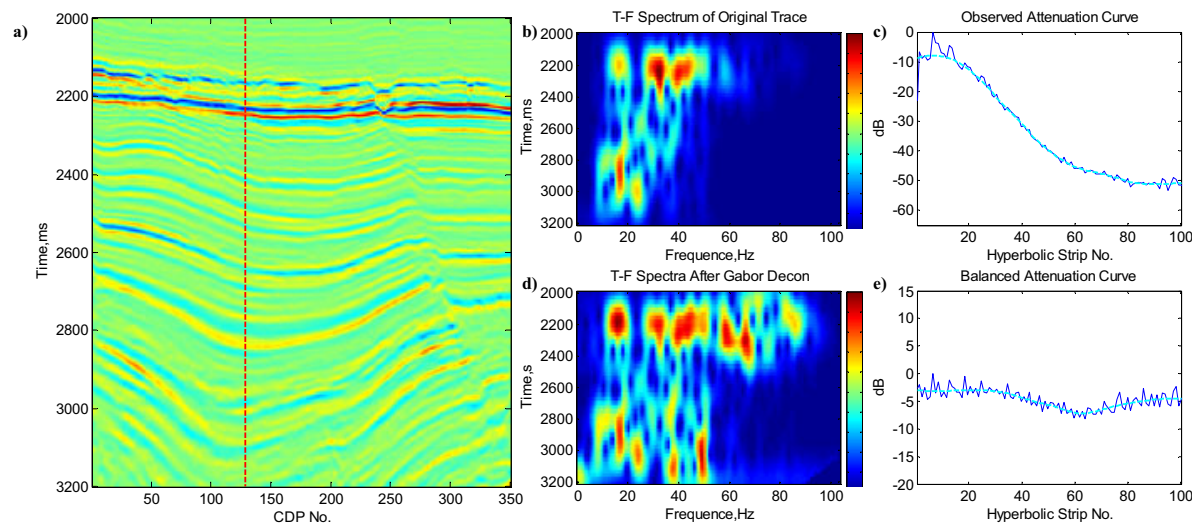

Fig. 5. A post-stack seismic section (a), original magnitude spectra of a field trace (b), and its observed attenuation curve (c), magnitude spectra of the same trace (d), and its attenuation curve (e) after Gabor deconvolution. Application of the improved Gabor deconvolution can significantly boost the deep spectra and well balance energy differences on the attenuation curve. 
In other words, nonstationarity can probably deviate us from the true answer if its influences are overlooked. In this example, reflectivity inversion method discussed here is constrained reflectivity inversion.

As mentioned above, the attenuation curve in log spectra can be taken as a quantitative measurement for nonstationarity. The curve is almost flat when seismic trace is free of nonstationarity, while a decreasing curve is a typical sign for the existence of nonstationarity. For the same seismic trace in Fig. 5a, its energy curve consists of RMS values from 100 hyperbolic bins in log spectra, showing as a decreasing curve (see Fig. 5c) which indicates that the seismic trace is actually under the influence of nonstationarity. Using log hyperbolic smoothing, we can remarkably boost the deep spectra (see Fig. 5d) and well balance energy differences on the attenuation curve (see Fig. 5e). Influence of nonstationarity can be regarded to be corrected during this process. In practice, nonstationarity is an overlooked issue that somehow has great effects on reflectivity estimation. Here, we would examine these effects on constrained reflectivity inversion. A comprehensive form of the inverse problem can be proposed as (Sacchi 1997, Canadas 2002, Velis 2008)

$$
\left(\boldsymbol{W}^{\prime} \boldsymbol{W}+\beta \boldsymbol{C}^{\prime} \boldsymbol{C}+\mu\left(\frac{1}{1+\left(\operatorname{diag}(\boldsymbol{r})^{*} \boldsymbol{r}\right) / 2 \sigma_{r}^{2}}\right)\right) \boldsymbol{r}=\boldsymbol{W}^{\prime} \boldsymbol{d}+\beta \boldsymbol{C}^{\prime} \boldsymbol{\xi}
$$

in which $\boldsymbol{d}, \boldsymbol{r}$, and $\boldsymbol{\xi}$, respectively, stand for the vector of seismic trace, reflectivity and acoustic impedance. Besides, $\boldsymbol{W}$ is the wavelet matrix and $\boldsymbol{C}$ denotes the causal matrix. In this regularized formula, $\sigma_{r}$ controls the sparseness of reflectivity, $\mu$ and $\beta$ are trade-off parameters for the sparseness constraint and impedance constraint.

If we apply constrained reflectivity inversion directly on the seismic section as most routines do, the obtained reflectivity information is given in the upper row in Fig. 6. To ensure a better view, results here are displayed according to its positive and negative reflectivity. As outlined in this figure, recovered reflectivity lacks lateral continuity, and ambiguities on reflectivity characterizations also exist. Actually, the recovered reflectivity is less reasonable because it somewhat conflicts with basic geologic settings of the study target. In contrast, if we investigate and balance the nonstationarity trace by trace beforehand, results of the constrained reflectivity inversion under the same constraints are obtained as the lower row of Fig. 6 shows. The estimated reflectivity is regarded to be much more reliable, for these ambiguities are significantly removed and the stable sedimentation features of the simple strata are better characterized.

In a routine manner, we directly apply constrained reflectivity inversion on our study area and then convolve the obtained reflectivity with a Ricker 

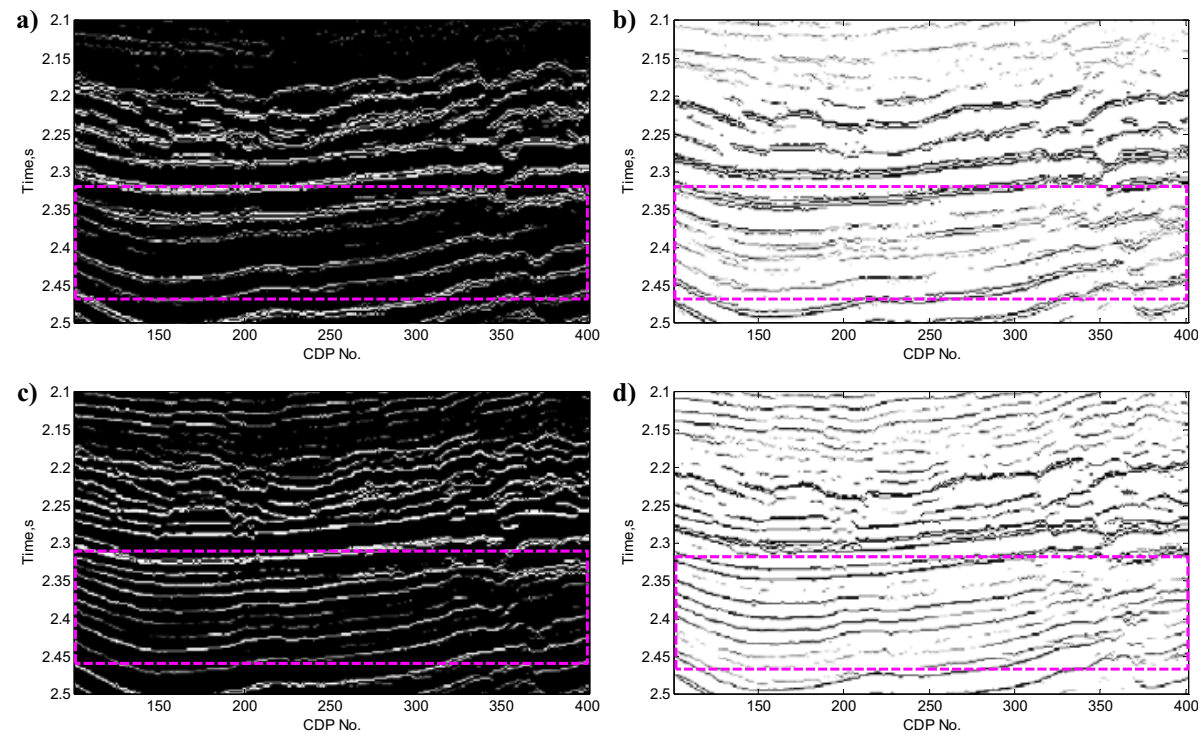

Fig. 6. Obtained positive reflection section (a), and negative reflection section (b) by conventional constrained reflectivity inversion without balancing nonstationarity, as well as improved positive reflection section (c), and negative reflection section (d) by correcting the influence of nonstationarity before performing constrained reflectivity inversion. Reliable reflectivity estimation is derived after correcting nonstationarity, thus leading to a better characterization of the stable sedimentation features in this area.
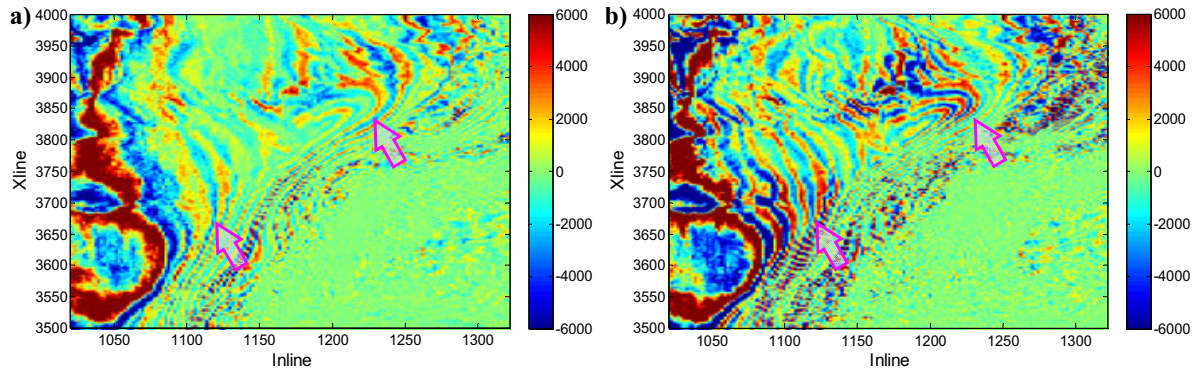

Fig. 7. Obtained amplitude time slice by traditional constrained reflectivity inversion (a), and that based on correcting the nonstationarity (b). Better definitions on reflection ambiguities are achieved after correcting the influence of nonstationarity with help of the improved Gabor deconvolution.

wavelet of a $60 \mathrm{~Hz}$ dominant frequency. As a result, extracted amplitude slice at $2350 \mathrm{~ms}$ is presented in Fig. 7a. In contrast, if we correct the influences of attenuation with the improved Gabor deconvolution prior to the reflectivity inversion and do the same convolution operation, the resultant 
amplitude slice is as Fig. $7 \mathrm{~b}$ shows. After correcting nonstationarity, it is evident that many ambiguities in Fig. 7 a are clearly characterized. Resultantly, better definitions on subsurface reflectivity information are achieved. This finding reminds us that certain amount of useful information may probably be veiled because of the influence of nonstationarity if we apply reflectivity inversion directly without giving enough considerations on the issue of non-stationarity.

\section{APPLICATIONS ON VARIANT- $Q$ ESTIMATION}

One major advantage of Gabor deconvolution lies in its capability in handling attenuation from a statistical perspective of energy, instead of knowing $Q$ value beforehand (Margrave et al. 2004). Considering the fact that $Q$ value is closely related to the gradient in Eq. 4, we can do get variant $Q$ estimations along the time axis by designing windows and doing linear fit over observed attenuation curve. In the following, we would discuss this $Q$ estimation method on complex carbonate reservoirs in Western China. Unlike conventional types of reservoir, the effective storage spaces are predominated by dissolved caves and fractures, which are usually developed with areal faults and water channels due to karstification.

Figure $8 \mathrm{a}$ is a post-stack seismic section which is obtained without any inappropriate AGC except a spherical spreading compensation by Kirchhoff pre-stack time migration. In this work, we use a 128-point Gaussian window
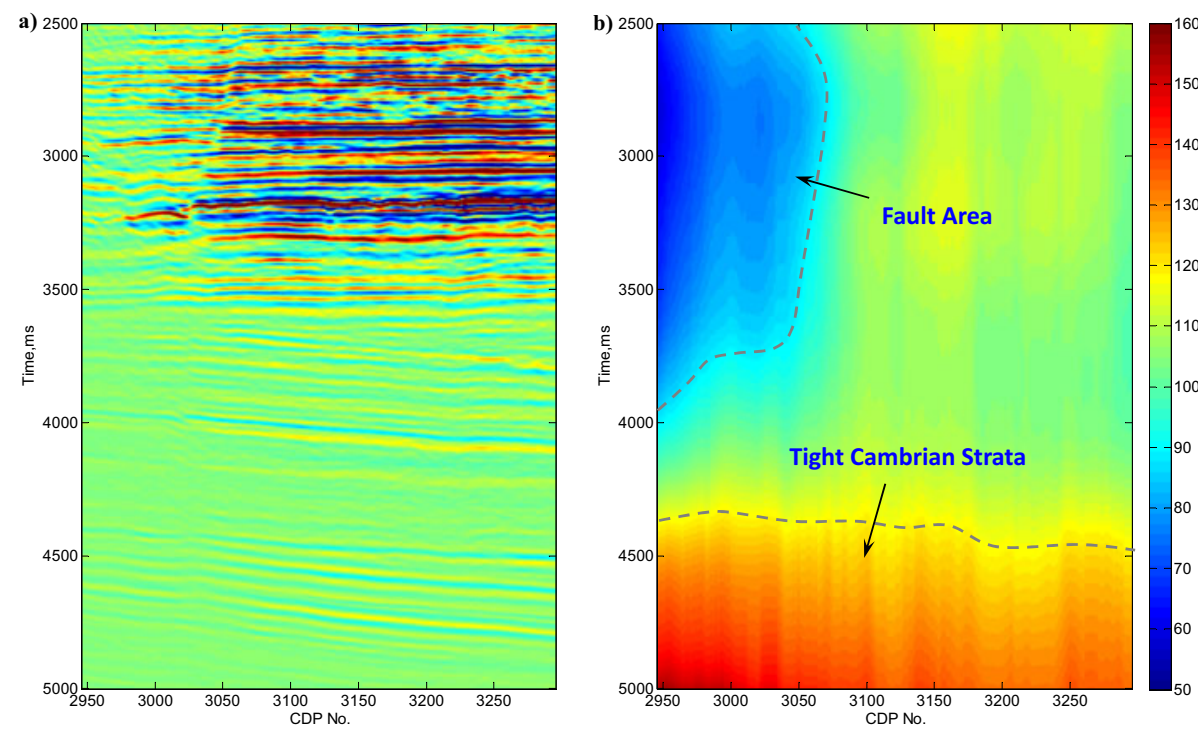

Fig. 8. Post-stack seismic section (a), and estimated $Q$-value section obtained by the improved Gabor deconvolution (b). Inferred $Q$ values show a degree of correlation with the basic geologies. 

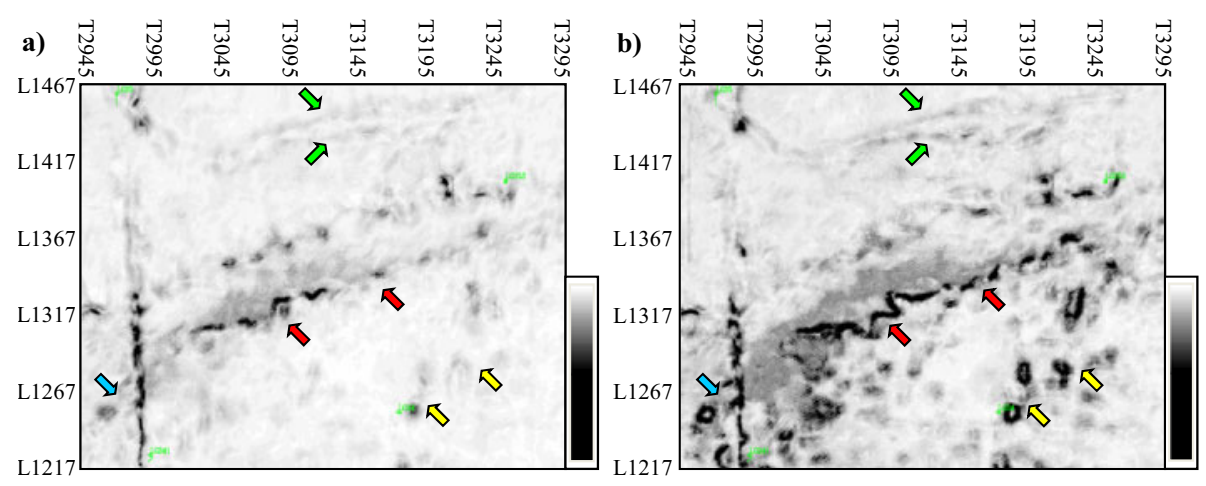

Fig. 9. Coherence attribute along target formation by traditional Kirchhoff pre-stack time migration (a), and $Q$-compensation migration (b). Based on proposed variant $Q$ estimation method, more geologic features (e.g., fault, water channel and pinch-out boundary) and dissolved caves are detected by using $Q$-compensation migration. Moreover, a closer relationship between the development of dissolved caves and geologic structures is also revealed.

with a 8-point step for Gabor transform, while the window for linear fit is designed to be 256-point in length and the whole log $t-f$ spectra is divided into 80 hyperbolic bins. After smoothing in both vertical and lateral directions, our estimated $Q$ section is shown in Fig. 8b. According to the geology of this area, there is a regional fault (i.e., the fault along the North-South direction in Fig. 9) accompanied with many secondary rifts across the left part of this section, where the induced strong attenuation should indicate low $Q$ values. Besides, tight Cambrian rocks at the bottom actually correspond to high $Q$ values. As a result, the inferred $Q$ values show a degree of correlation with these geologic phenomena.

Estimated $Q$ values can be further utilized to compensate amplitude and frequency loss caused by attenuation. Variant $Q$ values estimated by improved Gabor deconvolution can improve $Q$-compensation migration's performance. By applying these $Q$ values to $Q$-compensation migration (Bear et al. 2008), the obtained coherence attributes along target carbonate formation are given in Fig. 9. In Fig. 9b, more geologic features are more clearly revealed after $Q$-compensation migration, such as the major fault along the North-South direction and a pinch-out boundary in the Northeast-Southwest direction (outlined by red arrows), as well as the outline of water channel (outlined by green arrows). As for effective carbonate reservoirs (e.g., dissolved caves), they usually show themselves as "bead-like reflections" with strong amplitudes on seismic section, and can also be identified as strong spots on root-mean-square (i.e., RMS) amplitude or coherence maps (Yang et al. 2012). Consequently, more potential reservoirs are detected on the co- 
herence map obtained by $Q$-compensation migration that is accomplished based on variant $Q$ estimation. More importantly, discoveries in Fig. 9b are well supported by a more clear relationship between the dissolved caves and geologic structures (e.g., fault and water channel), which are necessarily needed for the implementation of karstification to form those dissolved caves.

\section{SUMMARY}

In log time-frequency spectra, we present an improved Gabor deconvolution based on a linear model for nonstationary convolution. To ensure a better factorization, we resort to a hyperbolic smoothing scheme which attaches importance on an iteration process to purify the estimation of attenuation. After removing the influence of attenuation, source wavelet can then be estimated by averaging along the time direction and smoothing along the frequency direction. As a matter of fact, the above procedures perform to factorize the nonstationary convolutional model efficiently. Because the separation in log spectra is largely done by subtractions instead of division and thus bypasses the frequent applications of pre-whitening factors, both accuracy and efficiency of this improved Gabor deconvolution are obviously improved. Applications on a numerical model and reef reservoir demonstrate that this improved method can further enrich the high frequency information and reveal more details with less computational expenses, when compared with the ordinary method.

Nonstationarity is a so common phenomenon that challenges the basic assumption of unchanging wavelet for most inversion methods which are built on Robinson convolution. Based on the improved Gabor deconvolution, the attenuation curve consisted of each hyperbolic bin's energy in log spectra can serve as a quantitative measurement on nonstationarity. The curve is almost flat when seismic trace is free of nonstationarity, while a decreasing curve is a typical sign for the existence of nonstationarity. Under this guidance, the nonstationarity can be regarded as basically corrected or balanced until the attenuation curve is almost flat. Joint applications with reflectivity inversion show that balancing nonstationarity well conditions seismic traces to the assumption of unchanging wavelet, thus can promote better characterizations for subsurface reflectivity. Otherwise, if we apply reflectivity inversion directly without giving enough considerations on nonstationarity, certain amount of useful reflectivity may probably be lost, causing ambiguities on reflectivity characterization.

Moreover, based on improved Gabor deconvolution, variant- $Q$ estimation can be achieved by making use of the gradient of this linear model (i.e., Eq. 4). And this estimation method largely depends on the statistical analysis of hyperbolic bin's energy in log spectra, and ought to be more robust. Ap- 
plication on complex carbonate reservoirs shows that estimated $Q$ values well agrees with the basic geologies of this area. And estimated variant $Q$ values can be further utilized to compensate amplitude and frequency loss caused by attenuation. In practice, based on proposed variant $Q$ estimation method, geologic features (e.g., fault, water channel, and pinch-out boundary) and dissolved caves are more clearly characterized by using $Q$ compensation migration. More importantly, those findings are well supported by a closer relationship between the dissolved caves and geologic structures (e.g., fault and water channel), which are necessarily needed for the implementation of karstification to form those dissolved caves.

Acknowledgements. This research is supported by Lab for Integration of Geology and Geophysics, China University of Petroleum and is funded by China National Nature Science Fund (2011CB201100). The authors are grateful for the sincere help offered by Mr. Hangyu Yue in $Q$-value correction and $Q$-compensation migration. Especially, the authors would like to express the sincere thanks to reviewers for their contributions to this paper.

\section{References}

Bear, L., J. Liu, and P. Traynin (2008), Efficient compensation for attenuation effects using pseudo Q migration. In: 78th SEG Annual Meeting, 9-14 November 2008, Las Vegas, USA, Society of Exploration Geophysicists, Expanded abstracts, SEG-2008-2206.

Bickel, S.H., and R.R. Natarajan (1985), Plane-wave Q deconvolution, Geophysics 50, 9, 1426-1439, DOI: 10.1190/1.1442011.

Canadas, G. (2002), A mathematical framework for blind deconvolution inverse problems. In: 72nd SEG Annual Meeting, 6-11 October 2002, Salt Lake City, USA, Society of Exploration Geophysicists, Expanded abstracts, SEG2002-2202.

Chen, Z., Y. Wang, X. Chen, and J. Li (2013), High-resolution seismic processing by Gabor deconvolution, J. Geophys. Eng. 10, 6, 065002, DOI: 10.1088/ 1742-2132/10/6/065002.

Griffiths, L.J., F.R. Smolka, and L.D. Trembly (1977), Adaptive deconvolution: A new technique for processing time-varying seismic data, Geophysics $\mathbf{4 2}$, 4, 742-759, DOI: 10.1190/1.1440743.

Grossman, J.P., G.F. Margrave, and M.P. Lamoureux (2002), Constructing adaptive, nonuniform Gabor frames from partitions of unity, CREWES Res. Rep. 14, $38,1-10$. 
Hargreaves, N.D., and A.J. Calvert (1991), Inverse Q filtering by Fourier transform, Geophysics 56, 4, 519-527, DOI: 10.1190/1.1443067.

Koehler, F., and M.T. Taner (1985), The use of the conjugate-gradient algorithm in the computation of predictive deconvolution operators, Geophysics 50, 12, 2752-2758, DOI: 10.1190/1.1441895.

Margrave, G.F. (1998), Theory of nonstationary linear filtering in the Fourier domain with application to time-variant filtering, Geophysics 63, 1, 244259, DOI: $10.1190 / 1.1444318$.

Margrave, G.F., and M.P. Lamoureux (2001), Gabor deconvolution, CREWES Res. Rep. 13, 18, 241-276.

Margrave, G.F., M.P. Lamoureux, J.P. Grossman, and V. Iliescu (2002), Gabor deconvolution of seismic data for source waveform and Q correction. In: 72nd SEG Annual Meeting, 6-11 October 2002, Salt Lake City, USA, Society of Exploration Geophysicists, Expanded abstracts, 2190-2193, DOI: 10.1190/1.1817142.

Margrave, G.F., D.C. Henley, M.P. Lamoureux, V. Iliescu, and J.P. Grossman (2003), Gabor deconvolution revisited. In: 73rd SEG Annual Meeting, 2631 October 2003, Dallas, USA, Society of Exploration Geophysicists, Expanded abstracts, 714-717, DOI: 10.1190/1.1818033.

Margrave, G.F., P.C. Gibson, J.P. Grossman, D.C. Henley, and M.P. Lamoureux (2004), Gabor deconvolution - theory and practice. In: 66th EAGE Conference and Exhibition, 7-10 June 2004, Paris, France, European Association of Geoscientists and Engineers.

Margrave, G.F., M.P. Lamoureux, and D.C. Henley (2011), Gabor deconvolution: Estimating reflectivity by nonstationary deconvolution of seismic data, Geophysics 76, 3, W15-W30, DOI: 10.1190/1.3560167.

Montana, C.A., and G.F. Margrave (2005), Phase correction in Gabor deconvolution. In: 75th SEG Annual Meeting, 6-11 November 2005, Houston, USA, Society of Exploration Geophysicists, Expanded abstracts, 2173-2176.

Robinson, E.A. (1954), Predictive decomposition of time series with applications to seismic exploration, Ph.D. Thesis, Massachusetts Institute of Technology, Cambridge, USA.

Sacchi, M.D.(1997), Reweighting strategies in seismic deconvolution, Geophys. J. Int. 129, 3, 651-656, DOI: 10.1111/j.1365-246X.1997.tb04500.x.

Sun, X., S.Z. Sun, X. Zhou, and W. Meng (2013), Gabor deconvolution based on hyperbolic smoothing in log spectra. In: 75th EAGE Conference and Exhibition incorporating SPE EUROPEC 2013, European Association of Geoscientists and Engineers, DOI: 10.3997/2214-4609.20130050.

Tria, M., M. van der Baan, A. Larue, and J.I. Mars (2007), Wavelet estimation and blind deconvolution of realistic synthetic seismic data by log spectral averaging. In: 77th SEG Annual Meeting, 23-26 September 2007, San Antonio, USA, Society of Exploration Geophysicists, Expanded abstracts, 19821986. 
Ulrych, T.J. (1971), Application of homomorphic deconvolution to seismology, Geophysics 36, 4, 650-660, DOI: 10.1190/1.1440202.

Velis, D.R. (2008), Stochastic sparse-spike deconvolution, Geophysics 73, 1, R1-R9, DOI: $10.1190 / 1.2790584$.

Wang, Y. (2002), A stable and efficient approach of inverse Q filtering, Geophysics 67, 2, 657-663, DOI: 10.1190/1.1468627.

Yang, P., S.Z. Sun, Y.L. Liu, H.Y. Li, G.J. Dan, and H.Q. Jia (2012), Origin and architecture of fractured-cavernous carbonate reservoirs and their influences on seismic amplitudes, The Leading Edge 31, 2, 140-150, DOI: 10.1190/1.3686911.

Yu, J., and D. Liu (2012), An application of structural filter and Gabor deconvolution for AVO processing. In: 82nd SEG Annual Meeting, 4-9 November 2012, Las Vegas, USA, Society of Exploration Geophysicists, Expanded abstracts, SEG-2012-1472.

Received 3 April 2014

Received in revised form 18 November 2014 Accepted 26 November 2014 